\title{
ON THE GROWTH OF LAPLACE-STIELTJES INTEGRALS
}

M. M. Sheremeta, A. O. Kuryliak. On the growth of Laplace-Stieltjes integrals, Mat. Stud. 50 (2018), 22-35.

In the paper it is investigated the growth of characteristics of Laplace-Stieltjes integrals $I(\sigma)=\int_{0}^{+\infty} f(x) d F(x)$, where $F$ is a nonnegative nondecreasing unbounded function continuous on the right on $[0,+\infty)$ and $f$ is a nonnegative on $[0,+\infty)$ function such that there exist $a \geq 0, b \geq 0$ and $h>0: \int_{x-a}^{x+b} f(t) d F(t) \geq h f(x)$ for all $x \geq a$. Assume that $\alpha, \beta$ are positive continuously differentiable functions increasing to $+\infty$ on $[0,+\infty)$ such that: a) $\alpha(c x)=(1+o(1)) \alpha(x)(x \rightarrow+\infty)$ for any $c>0$; b) $\beta(x(1+o(1)))=(1+o(1)) \beta(x)$ $(x \rightarrow+\infty)$; c) $\frac{d \beta^{-1}(\alpha(x) / \varrho)}{d \ln x}=O(1)(x \rightarrow+\infty)$ for every $\varrho \in(0,+\infty)$. The main results of the paper are contained in Theorems 5 and 7 and are derived from the following two statements of independent interest. If $F$ satisfies condition $\ln F(x)=o\left(x \beta^{-1}\left(\frac{\alpha(x)}{\varrho}\right)\right)(x \rightarrow+\infty)$, then $\varrho_{\alpha \beta}(I)=k_{\alpha \beta}(f)$ (Theorem 1). If in additional the function $v(x)=-(\ln f(x))^{\prime}$ is continuous and increasing on $\left[x_{0},+\infty\right)$ and $\varrho_{\alpha \beta}(I)<+\infty$, then $\lambda_{\alpha \beta}(I)=\varkappa_{\alpha \beta}(f)$ (Theorem 2), where

$$
\varlimsup_{\sigma \rightarrow+\infty} \frac{\alpha(\ln I(\sigma))}{\beta(\sigma)}:=\left\{\begin{array}{l}
\varrho_{\alpha \beta}(I), \\
\lambda_{\alpha \beta}(I),
\end{array} \quad \underline{\varlimsup_{\rightarrow+\infty}} \frac{\alpha(x)}{\beta\left(\frac{1}{x} \ln \frac{1}{f(x)}\right)}:=\left\{\begin{array}{l}
k_{\alpha \beta}(f), \\
\varkappa_{\alpha \beta}(f) .
\end{array}\right.\right.
$$

Similar results are proved also for so called the modified generalized order and lower order.

1. Introduction. For an entire function

$$
f(z)=\sum_{n=0}^{\infty} a_{n} z^{n}
$$

let $\varrho(f)$ be its order and $\sigma(f)$ be its type. Using Hadamard's formulas fo the finding of these characteristics, E.G. Calys ([1]) proved the following theorems.

Theorem A. Suppose that entire functions $f_{1}(z)=\sum_{n=0}^{\infty} a_{n, 1} z^{n}$ and $f_{2}(z)=\sum_{n=0}^{\infty} a_{n, 2} z^{n}$ have finite orders and regular growth (in sense of the equality of order $\varrho[f]$ and lower order $\varrho[f])$ and the sequences $\left(\left|a_{n, 1} / a_{n+1,1}\right|\right)$ and $\left(\left|a_{n, 2} / a_{n+1,2}\right|\right)$ are non-decreasing for $n \geq n_{0}$. If

$$
\ln \left(1 /\left|a_{n}\right|\right)=(1+o(1)) \sqrt{\ln \left(1 /\left|a_{n, 1}\right|\right) \ln \left(1 /\left|a_{n, 2}\right|\right)}, \quad n \rightarrow \infty
$$

then the function $f$ has regular growth and $\varrho[f]=\sqrt{\varrho\left[f_{1}\right] \varrho\left[f_{2}\right]}$.

2010 Mathematics Subject Classification: 30B50, 30D10, 30D15.

Keywords: Laplace-Stieltjes integral; regular growth; asymptotic estimate. doi:10.15330/ms.50.1.22-35

(C) M. M. Sheremeta, A. O. Kuryliak, 2018 
Theorem B. Suppose that functions $f_{1}$ and $f_{2}$ from Theorem $A$ have the same order $\varrho\left[f_{1}\right]=\varrho\left[f_{2}\right]=\varrho \in(0,+\infty)$ and types $\sigma\left[f_{1}\right]=\sigma_{1}, \sigma\left[f_{2}\right]=\sigma_{2}$. Also suppose that $a_{n, 1} \neq 0$ and $\left|a_{n, 2}\right| \geq\left|a_{n, 1}\right| / l\left(1 /\left|a_{n, 1}\right|\right)$ for all $n \geq n_{0}$, where $l$ is a slowly varying function. If

$$
\left|a_{n}\right|=(1+o(1)) \sqrt{\left|a_{n, 1}\right|\left|a_{n, 2}\right|}, \quad n \rightarrow \infty,
$$

then the function $f$ has order $\varrho[f]=\varrho$ and type $\sigma[f] \leq \sqrt{\sigma_{1} \sigma_{1}}$.

We remark that R.S.L. Srivastava ([2,3]) tried to prove Theorem A without assumptions $a_{n, 1} \neq 0$ and $\left|a_{n, 2}\right| \geq\left|a_{n, 1}\right| / l\left(1 /\left|a_{n, 1}\right|\right)$ for all $n \geq n_{0}$ and Theorem $\mathrm{B}$ without condition of the nondecrease of the sequences $\left(\left|a_{n, 1} / a_{n+1,1}\right|\right)$ and $\left(\left|a_{n, 2} / a_{n+1,2}\right|\right)$. On the fallaciousness of such statements it was indicated in Math. Rev., 1963, V.25, №2204, №2206.

In [4] Theorems A and B are transfered on entire Dirichlet series. Here we will obtain such theorems for Laplace-Stieltjes integrals.

Let $V$ be the class of which are nonnegative nondecreasing unbounded and continuous on the right functions $F$ on $[0,+\infty)$.

The Laplace-Stieltjes transform of a real-valued function $g$ is given, usually, by a Lebesgue-Stieltjes integral of the form $\int_{0}^{+\infty} e^{z x} d g(x)$. We write this transformation in a different form. For a nonnegative function $f$ on $[0,+\infty)$ the integral

$$
I(\sigma)=\int_{0}^{\infty} f(x) e^{x \sigma} d F(x), \quad \sigma \in \mathbb{R},
$$

is called of Laplace-Stieltjes ( [5-7]). Integral (1) is a direct generalization of the ordinary Laplace integral $I(\sigma)=\int_{0}^{\infty} f(x) e^{x \sigma} d x$ and of the Dirichlet series $D(\sigma)=\sum_{n=0}^{\infty} a_{n} e^{\lambda_{n} \sigma}$ with nonnegative coefficients $a_{n}$ and exponents $\lambda_{n}, 0 \leq \lambda_{n} \uparrow+\infty(n \rightarrow \infty)$, if we choose $F(x)=n(x)=\sum_{\lambda_{n} \leq x} 1$ and $f\left(\lambda_{n}\right)=a_{n} \geq 0$ for all $n \geq 0$ (see also [5,8]).

Let

$$
\mu(\sigma)=\mu(\sigma, I)=\max \left\{f(x) e^{x \sigma}: x \geq 0\right\}, \quad \sigma \in \mathbb{R},
$$

be the maximum of the integrand, $\sigma_{c}$ be the abscissa of convergence of the integral (1) and $\sigma_{\mu}$ be the abscissa of maximum of the integrand. Then $([7, \mathrm{p} .8])$

$$
\sigma_{\mu}=\varliminf_{x \rightarrow+\infty} \frac{1}{x} \ln \frac{1}{f(x)}
$$

and if either $\ln F(x)=o(x)$ or $\ln F(x)=o(\ln f(x))$ as $x \rightarrow+\infty$ then $([7, \mathrm{p} .13]) \sigma_{c} \leq \sigma_{\mu}$. Also we remark that if $\ln F(x)=O(x)$ as $x \rightarrow+\infty$ and $\sigma_{\mu}=+\infty$ then ([7, p.11]) $\sigma_{c}=+\infty$.

To obtain the inequality $\sigma_{c} \geq \sigma_{\mu}$ we introduce as in [7, p.21] the concept of a regular variation of $f$ in regard to $F$. We say that a positive function $f$ has regular variation in regard to $F$ if there exist $a \geq 0, b \geq 0$ and $h>0$ such that for all $x \geq a$

$$
\int_{x-a}^{x+b} f(t) d F(t) \geq h f(x) .
$$

Then [7, p.21] if $F \in V$ and $f$ has regular variation in regard to $F$ then $\sigma_{c} \leq \sigma_{\mu}$. Thus, if $F \in V$ and $f$ has regular variation in regard to $F$ and either $\ln F(x)=o(x)$ or $\ln F(x)=$ $o(\ln f(x))$ as $x \rightarrow+\infty$ then $\sigma_{c}=\sigma_{\mu}$. 
Further we assume that $\sigma_{c}=\sigma_{\mu}=+\infty$.

2. Generalized orders. Let $L$ be the class of continuous increasing functions $\alpha$ such that $\alpha(x) \geq 0$ for $x \geq x_{0}, \alpha(x)=\alpha\left(x_{0}\right)$ for $x \leq x_{0}$, and on $\left[x_{0},+\infty\right)$ the function $\alpha$ increases to $+\infty$. We say that $\alpha \in L^{0}$ if $\alpha \in L$ and $\alpha(x(1+o(1)))=(1+o(1)) \alpha(x)$ as $x \rightarrow+\infty$; further, $\alpha \in L_{s i}$ if $\alpha \in L$ and for any $c>0 \alpha(c x)=(1+o(1)) \alpha(x)$ as $x \rightarrow+\infty$. It is easy to see that $L_{s i} \subset L^{0}$. Functions from $L_{s i}$ are called slowly increasing. In future we will need the next lemma [9].

Lemma 1. Let $\beta \in L$ and

$$
B(\delta)=\varlimsup_{x \rightarrow+\infty} \frac{\beta((1+\delta) x)}{\beta(x)}, \quad \delta>0 .
$$

Then in order that $\beta \in L^{0}$ it is necessary and sufficient that $B(\delta) \rightarrow 1$ as $\delta \rightarrow 0$.

Let $\alpha \in L, \beta \in L$, and $G$ be an arbitrary function on $\left[\sigma_{0},+\infty\right)$. The value

$$
\varrho_{\alpha \beta}(G)=\varlimsup_{\sigma \rightarrow+\infty} \frac{\alpha(G(\sigma))}{\beta(\sigma)}
$$

is called a generalized order of $G$. If we choose $G(\sigma)=\ln I(\sigma)$ then from (2) we obtain the definition of the generalized order $\varrho_{\alpha \beta}(I)$ of the Laplace-Stieltjes integral (1). Also define

$$
k_{\alpha \beta}(f)=\varlimsup_{x \rightarrow+\infty} \frac{\alpha(x)}{\beta\left(\frac{1}{x} \ln \frac{1}{f(x)}\right)} .
$$

First we remark that if the functions $\alpha \in L^{0}$ and $\beta \in L^{0}$ are continuously differentiable and for every $\varrho \in(0,+\infty)$

$$
\frac{d \beta^{-1}(\alpha(x) / \varrho)}{d \ln x}=O(1), \quad x \rightarrow+\infty,
$$

then $([7, \mathrm{p} .77]) \varrho_{\alpha \beta}(\ln \mu)=k_{\alpha \beta}(f)$, and if for every $\varrho \in(0,+\infty)$

$$
\ln F(x)=o\left(x \beta^{-1}\left(\frac{\alpha(x)}{\varrho}\right)\right), \quad x \rightarrow+\infty,
$$

then $([7, \mathrm{p} .77]) \varrho_{\alpha \beta}(I) \leq \varrho_{\alpha \beta}(\ln \mu)$. On the other hand, if $f$ has a regular variation in regard to $F$ then $([7, \mathrm{p} .81]) \varrho_{\alpha \beta}(I) \geq \varrho_{\alpha \beta}(\ln \mu)$ for each $\alpha \in L^{0}$ and $\beta \in L$.

Thus, the following theorem is true.

Theorem 1. Let $F \in V, f$ have regular variation in regard to $F$ and functions $\alpha \in L_{s i}$ and $\beta \in L^{0}$ satisfy condition (3). If $F$ satisfies condition (4) then $\varrho_{\alpha \beta}(I)=k_{\alpha \beta}(f)$.

Now we put

$$
\lambda_{\alpha \beta}(I)=\varliminf_{\sigma \rightarrow+\infty} \frac{\alpha(\ln I(\sigma))}{\beta(\sigma)}, \quad \lambda_{\alpha \beta}(\ln \mu)=\varliminf_{\sigma \rightarrow+\infty} \frac{\alpha(\ln \mu(\sigma))}{\beta(\sigma)}, \quad \varkappa_{\alpha \beta}(f)=\varliminf_{x \rightarrow+\infty} \frac{\alpha(x)}{\beta\left(\frac{1}{x} \ln \frac{1}{f(x)}\right)} .
$$

Proposition 1. If $\alpha \in L$ and $\beta \in L^{0}$ then $\lambda_{\alpha \beta}(\ln \mu) \geq \varkappa_{\alpha \beta}(f)$. 
Indeed, if $\varkappa_{\alpha \beta}(f)>0$ then for each $\varkappa \in\left(0, \varkappa_{\alpha \beta}(f)\right)$ and all $x \geq x_{0}=x_{0}(\varkappa)$ we have $\left.\ln f(x) \geq-x \beta^{-1}(\alpha(x) / \varkappa)\right)$. Therefore, $\left.\ln \mu(\sigma) \geq-x \beta^{-1}(\alpha(x) / \varkappa)\right)+x \sigma$ for all $\sigma$ and $x \geq x_{0}$. Choosing $x=\alpha^{-1}(\varkappa \beta(\sigma-1)) \geq x_{0}$ for $\sigma \geq \sigma_{0}$ hence we obtain

$$
\ln \mu(\sigma) \geq a^{-1}(\varkappa \beta(\sigma-1))=a^{-1}(\varkappa(1+o(1)) \beta(\sigma)), \quad \sigma \rightarrow+\infty .
$$

Therefore, $\lambda_{\alpha \beta}(\ln \mu) \geq \varkappa$ and in view of the arbitrariness of $\varkappa$ we have $\lambda_{\alpha \beta}(\ln \mu) \geq \varkappa_{\alpha \beta}(f)$. If $\varkappa_{\alpha \beta}(f)=0$ this inequality is obvious.

Proposition 2. Let $\alpha \in L_{s i}, \beta \in L^{0}$ and condition (3) hold. If the function $v(x)=$ $-(\ln f(x))^{\prime}$ is continuous and increasing on $\left[x_{0},+\infty\right)$ then $\lambda_{\alpha \beta}(\ln \mu) \leq \varkappa_{\alpha \beta}(f)$.

Indeed, since $v(x)=-(\ln f(x))^{\prime}$ is continuous and increasing on $\left[x_{0},+\infty\right)$, the function $\ln f(x)+\sigma x$ has the unique point $x$ of the maximum such that $\sigma=v(x)$, and $\ln \mu(\sigma)=$ $\ln f(x)+\sigma x$, where $\sigma=v(x)$.

Suppose that $\varkappa_{\alpha \beta}(f)<+\infty$. Then for every $\varkappa>\varkappa_{\alpha \beta}(f)$ there exists a sequence $\left(x_{k}\right) \uparrow$ $+\infty$ such that $\left.\ln f\left(x_{k}\right) \leq-x_{k} \beta^{-1}\left(\alpha\left(x_{k}\right) / \varkappa\right)\right)$. We put $\mu^{*}(\sigma)=f\left(x_{k}\right) e^{\sigma x_{k}}$. Since $\mu(\sigma)=$ $f(x) e^{\sigma x}$ for $\sigma=v^{-1}(x)$ we have $\mu\left(\sigma_{k}\right)=\mu^{*}\left(\sigma_{k}\right)$ for $\sigma_{k}=v\left(x_{k}\right)$. Hence

$$
\ln \mu\left(\sigma_{k}\right)=\ln \mu^{*}\left(\sigma_{k}\right) \leq \max _{k}\left\{-x_{k} \beta^{-1}\left(\alpha\left(x_{k}\right) / \varkappa\right)+x_{k} \sigma_{k}\right\} \leq \max _{x}\left\{-x \beta^{-1}(\alpha(x) / \varkappa)+x \sigma_{k}\right\} .
$$

In view of $(3)$

$$
\begin{gathered}
\left.\left.\left(-x \beta^{-1}(\alpha(x) / \varkappa)\right)+x \sigma_{k}\right)^{\prime}=-\beta^{-1}(\alpha(x) / \varkappa)\right)-\frac{d \beta^{-1}(\alpha(x) / k)}{d \ln x}+\sigma_{k}= \\
\left.=-\beta^{-1}(\alpha(x) / \varkappa)\right)+\sigma_{k}+O(1), \quad x \rightarrow+\infty,
\end{gathered}
$$

i. e. the function $\left.-x \beta^{-1}(\alpha(x) / \varkappa)\right)+x \sigma_{k}$ attains its maximum at the point

$$
\begin{gathered}
x\left(\sigma_{k}\right)=\alpha^{-1}\left(\varkappa \beta\left(\sigma_{k}+O(1)\right)\right), x \rightarrow+\infty, \\
\left.\left.\ln \mu\left(\sigma_{k}\right) \leq-\alpha^{-1}\left(\varkappa \beta\left(\sigma_{k}+O(1)\right)\right)\left(\sigma_{k}+O(1)\right)\right)\right)+\sigma_{k} \alpha^{-1}\left(\varkappa \beta\left(\sigma_{k}+O(1)\right)\right)= \\
=O\left(\alpha^{-1}\left(\varkappa \beta\left(\sigma_{k}+O(1)\right)\right)\right), \quad k \rightarrow \infty .
\end{gathered}
$$

Since $\alpha \in L_{s i}$ and $\beta \in L^{0}$, hence it follows that $\lambda_{\alpha \beta}(\ln \mu) \leq \varkappa$. In view of the arbitrariness of $\varkappa$ we have $\lambda_{\alpha \beta}(\ln \mu) \leq \varkappa_{\alpha \beta}(f)$. If $\varkappa_{\alpha \beta}(f)=+\infty$ this inequality is obvious.

Proposition 3. If $\alpha \in L^{0}, \beta \in L$ and $f$ has regular variation in regard to $F$ then $\lambda_{\alpha \beta}(\ln \mu) \leq$ $\lambda_{\alpha \beta}(I)$.

Indeed, if $f$ has regular variation in regard to $F$ then $([7, \mathrm{p} .75])$

$$
\ln \mu(\sigma) \leq(1+o(1)) \ln I(\sigma), \quad \sigma \rightarrow+\infty,
$$

whence $\lambda_{\alpha \beta}(\ln \mu) \leq \lambda_{\alpha \beta}(I)$.

Proposition 4. Let the functions $\alpha \in L^{0}$ and $\beta \in L^{0}$ satisfy condition (3), and the function $F \in V$ satisfies condition (4). If $\varrho_{\alpha \beta}(\ln \mu)<+\infty$ then $\lambda_{\alpha \beta}(\ln \mu) \geq \lambda_{\alpha \beta}(I)$. 
Indeed, since $\varrho_{\alpha \beta}(\ln \mu)<+\infty$, we have $k_{\alpha \beta}(f)=\varrho_{\alpha \beta}(\ln \mu)<+\infty$, that is $\ln f(x) \leq$ $\left.-x \beta^{-1}(\alpha(x) / k)\right)$ for some $k<+\infty$ and in view of (4) $\varlimsup_{x \rightarrow+\infty} \frac{\ln F(x)}{\ln (1 / f(x))}=0$. Therefore, [7, p.61]

$$
I(\sigma) \leq K(\varepsilon) \mu(\sigma /(1-\varepsilon))^{1-\varepsilon}
$$

for every $\varepsilon \in(0,1)$ and all $\sigma \geq \sigma_{0}(\varepsilon)$. Hence,

$$
\lambda_{\alpha \beta}(I) \leq \lambda_{\alpha \beta}(\ln \mu) \varlimsup_{\sigma \rightarrow+\infty} \frac{\beta(\sigma /(1-\varepsilon))}{\beta(\sigma)} .
$$

Since $\beta \in L^{0}$ by Lemma $1 \varlimsup_{x \rightarrow+\infty} \frac{\beta(\sigma /(1-\varepsilon))}{\beta(\sigma)} \rightarrow 1$ as $\varepsilon \rightarrow 0$. Thus, $\lambda_{\alpha \beta}(I) \leq \lambda_{\alpha \beta}(\ln \mu)$.

Combining Propositions 1-4 we get the following theorem.

Theorem 2. Let functions $\alpha \in L_{s i}$ and $\beta \in L^{0}$ satisfy condition (3) and the function $F \in V$ satisfy condition (4). Suppose that the function $f$ has a regular variation in regard to $F$ and $v(x)=-(\ln f(x))^{\prime}$ is continuous and increasing on $\left[x_{0},+\infty\right)$. If $\varrho_{\alpha \beta}(I)<+\infty$ then $\lambda_{\alpha \beta}(I)=\varkappa_{\alpha \beta}(f)$.

3. Modified generalized orders. The values

$$
\varrho_{\alpha \beta}^{M}(I)=\varlimsup_{\sigma \rightarrow+\infty} \frac{1}{\beta(\sigma)} \alpha\left(\frac{\ln I(\sigma)}{\sigma}\right), \quad \lambda_{\alpha \beta}^{M}(I)=\varliminf_{\sigma \rightarrow+\infty} \frac{1}{\beta(\sigma)} \alpha\left(\frac{\ln I(\sigma)}{\sigma}\right)
$$

are called the modified generalized order and the modified lower generalized order of $I$, respectively. If in $(7)$ we choose $\ln \mu(\sigma)$ instead $I(\sigma)$ then we obtain definitions of $\varrho_{\alpha \beta}^{M}(\ln \mu)$ and $\lambda_{\alpha \beta}^{M}(\ln \mu)$.

Proposition 5. Let either $\alpha \in L_{\text {si }}$ and $\beta \in L^{0}$ or $\alpha \in L^{0}$ and $\beta \in L_{\text {si }}$, and the function $F \in V$ satisfies condition (4). Then $\varrho_{\alpha \beta}^{M}(\ln \mu)=k_{\alpha \beta}(f)$.

Proof. Suppose that $\varrho_{\alpha \beta}^{M}(\ln \mu)<+\infty$. Then for every $\varrho>\varrho_{\alpha \beta}^{M}(\ln \mu)$, all $\sigma \geq \sigma_{0}(\varrho)$ and $x \geq 0$ we obtain $\ln f(x)+\sigma x \leq \ln \mu(\sigma) \leq \sigma \alpha^{-1}(\varrho \beta(\sigma))$, that is $\ln f(x) \leq \sigma \alpha^{-1}(\varrho \beta(\sigma))-\sigma x$. We choose $\sigma=\sigma(x)=\beta^{-1}(\alpha(\delta x) / \varrho)$ for an arbitrary $\delta \in(0,1)$. Then $\sigma(x) \geq \sigma_{0}(\varrho)$ for $x \geq x_{0}=x_{0}(\varrho, \delta)$ and $\ln f(x) \leq-(1-\delta) x \beta^{-1}(\alpha(\delta x) / \varrho)$ for $x \geq x_{0}$, whence

$$
\begin{gathered}
k_{\alpha \beta}(f)=\varlimsup_{x \rightarrow+\infty} \frac{\alpha(x)}{\beta\left(\frac{1}{x} \ln \frac{1}{f(x)}\right)}=\varlimsup_{x \rightarrow+\infty}\left(\frac{\alpha(\delta x)}{\beta\left(\frac{1}{(1-\delta) x} \ln \frac{1}{f(x)}\right)} \frac{\beta\left(\frac{1}{(1-\delta) x} \ln \frac{1}{f(x)}\right)}{\beta\left(\frac{1}{x} \ln \frac{1}{f(x)}\right)}\right) \frac{\alpha(x)}{\alpha(\delta x)} \leq \\
\leq \varrho \varlimsup_{x \rightarrow+\infty} \frac{\beta(x /(1-\delta))}{\beta(x)} \varlimsup_{x \rightarrow+\infty} \frac{\alpha(x)}{\alpha(\delta x)} .
\end{gathered}
$$

If $\alpha \in L_{s i}$ and $\beta \in L^{0}$ then $\varlimsup_{x \rightarrow+\infty} \frac{\alpha(x)}{\alpha(\delta x)}=1$ and by Lemma $1 \varlimsup_{x \rightarrow+\infty} \frac{\beta(x /(1-\delta))}{\beta(x)} \rightarrow 1$ as $\delta \rightarrow 0$. Hence in view of the arbitrariness of $\varrho$ we obtain

$$
k_{\alpha \beta}(f) \leq \varrho_{\alpha \beta}^{M}(\ln \mu) .
$$

If $\beta \in L_{s i}$ and $\alpha \in L^{0}$ then $\varlimsup_{x \rightarrow+\infty} \frac{\beta(x /(1-\delta))}{\beta(x)}=1$, and by Lemma $1 \varlimsup_{x \rightarrow+\infty} \frac{\alpha(x)}{\alpha(\delta x)} \rightarrow 1$ as $\delta \rightarrow 1$, and we again obtain inequality (9). If $\varrho_{\alpha \beta}^{M}(\ln \mu)=+\infty$ inequality (9) is obvious. 
Now assume that $k_{\alpha \beta}(f) \neq \varrho_{\alpha \beta}^{M}(\ln \mu)$. Then in view of $(8) k_{\alpha \beta}(f)<\varrho_{\alpha \beta}^{M}(\ln \mu)$ and if we choose $k_{\alpha \beta}(f)<\varrho<\varrho_{\alpha \beta}^{M}(\ln \mu)$ then $\left.\ln f(x) \leq-x \beta^{-1}(\alpha(x) / \varrho)\right)$ for $x \geq x_{0}(\varrho)$, i. e.

$$
\begin{gathered}
\left.\ln \mu(\sigma) \leq \max \left\{\max _{x \leq x_{0}(\varrho)}(\ln f(x)+x \sigma), \max _{x \geq x_{0}(\varrho)}\left(-x \beta^{-1}(\alpha(x) / \varrho)\right)+x \sigma\right)\right\} \leq \\
\leq \max _{x \geq 0}\left(x\left(\sigma-\beta^{-1}(\alpha(x) / \varrho)\right)\right)+O(\sigma), \quad \sigma \rightarrow+\infty .
\end{gathered}
$$

Since $\ln \mu(\sigma) \rightarrow+\infty$ as $\sigma \rightarrow+\infty$, the function $x\left(\sigma-\beta^{-1}(\alpha(x) / \varrho)\right)$ attains the maximum at the point $x=x(\sigma)$ such that $\sigma-\beta^{-1}(\alpha(x) / \varrho)>0$, that is $x(\sigma) \leq \alpha^{-1}(\varrho \beta(\sigma))$. Therefore, $\ln \mu(\sigma) \leq x(s)\left(\sigma-\beta^{-1}(\alpha(x(\sigma)) / \varrho)\right)+O(\sigma) \leq \sigma x(\sigma)+O(\sigma) \leq \sigma \alpha^{-1}(\varrho \beta(\sigma))+O(\sigma), \quad \sigma \rightarrow+\infty$, whence it follows that $\varrho_{\alpha \beta}^{M}(\ln \mu) \leq \varrho$. It is a contradiction that therefore, Proposition 5 is proved.

Proposition 6. Let $\alpha \in L^{0}, \beta \in L^{0}$, and $f$ have regular variation in regard to $F$. Then $\varrho_{\alpha \beta}^{M}(\ln \mu)=\varrho_{\alpha \beta}^{M}(I)$.

Indeed, if $f$ has regular variation in regard to $F$ then from (5) we obtain $\varrho_{\alpha \beta}^{M}(\ln \mu) \leq$ $\leq \varrho_{\alpha \beta}^{M}(I)$. On the other hand, if $\varrho_{\alpha \beta}^{M}(\ln \mu)<+\infty$ then in view of Proposition 5 , as in the proof of Proposition 4, we have (6) for every $\varepsilon \in(0,1)$ and all $\sigma \geq \sigma_{0}(\varepsilon)$. Since $\alpha \in L^{0}$ and $\beta \in L^{0}$, we have $\varrho_{\alpha \beta}^{M}(I) \leq \varrho_{\alpha \beta}^{M}(\ln \mu)$. Thus, $\varrho_{\alpha \beta}^{M}(\ln \mu)=\varrho_{\alpha \beta}^{M}(I)$.

Uniting Propositions 5 and 6 we get the following theorem.

Theorem 3. Let either $\alpha \in L_{s i}$ and $\beta \in L^{0}$ or $\alpha \in L^{0}$ and $\beta \in L_{s i}$, the function $F \in V$ satisfies condition (4) and $f$ has regular variation in regard to $F$. Then $\varrho_{\alpha \beta}^{M}(I)=k_{\alpha \beta}(f)$.

Proposition 7. Let either $\alpha \in L_{s i}$ and $\beta \in L^{0}$ or $\alpha \in L^{0}$ and $\beta \in L_{s i}$. Then $\lambda_{\alpha \beta}^{M}(\ln \mu) \geq$ $\varkappa_{\alpha \beta}(f)$. Moreover, if $v(x)=-(\ln f(x))^{\prime}$ is continuous and increasing on $\left[x_{0},+\infty\right)$ then $\lambda_{\alpha \beta}^{M}(\ln \mu)=\varkappa_{\alpha \beta}(f)$.

Indeed, if $\varkappa_{\alpha \beta}(f)>0$ then for each $\varkappa \in\left(0, \varkappa_{\alpha \beta}(f)\right)$ and all $x \geq x_{0}=x_{0}(\varkappa)$ as in the proof of Proposition 7 we obtain $\left.\ln \mu(\sigma) \geq-x \beta^{-1}(\alpha(x) / \varkappa)\right)+x \sigma$ for all $\sigma$ and $x \geq x_{0}$. Choosing $x=\alpha^{-1}(\varkappa \beta(\delta \sigma))$, where $0<\delta<1$, we have $\ln \mu(\sigma) \geq(1-\delta) \sigma a^{-1}(\varkappa \beta(\delta \sigma))$. Hence

$$
\lambda_{\alpha \beta}^{M}(\ln \mu) \geq \varliminf_{\sigma \rightarrow+\infty} \frac{\alpha\left((1-\delta) a^{-1}(\varkappa \beta(\delta \sigma))\right)}{\beta(\sigma)}
$$

If $\alpha \in L_{s i}$ and $\beta \in L^{0}$ hence we have as above $\lambda_{\alpha \beta}^{M}(\ln \mu) \geq \varkappa \varliminf_{\sigma \rightarrow+\infty}^{\lim } \frac{\beta(\delta \sigma)}{\beta(\sigma)} \rightarrow \varkappa$ as $\delta \rightarrow 1$. If $\alpha \in L^{0}$ and $\beta \in L_{s i}$ then

$$
\lambda_{\alpha \beta}^{M}(\ln \mu) \geq \varkappa \varliminf_{\sigma \rightarrow+\infty} \frac{\alpha\left((1-\delta) a^{-1}(\varkappa \beta(\delta \sigma))\right)}{\varkappa \beta(\delta \sigma)} \frac{\beta(\delta \sigma)}{\beta(\sigma)}=\varkappa \varliminf_{t \rightarrow+\infty} \frac{\alpha\left((1-\delta) a^{-1}(t)\right.}{t} \rightarrow \varkappa
$$

as $\delta \rightarrow 0$. Therefore, $\lambda_{\alpha \beta}^{M}(\ln \mu) \geq \varkappa_{\alpha \beta}(f)$.

On the other hand, as in the proof Proposition 2 we obtain $\ln \mu(\sigma)=\ln f(x)+\sigma x$ for $\sigma=v(x)$. Supposing that $\varkappa_{\alpha \beta}(f)<+\infty$, for $\varkappa>\varkappa_{\alpha \beta}(f)$ and some sequence $\left(x_{k}\right) \uparrow+\infty$ as in the proof of Proposition 2 we obtain for $\sigma_{k}=v\left(x_{k}\right)$

$$
\left.\left.\ln \mu\left(\sigma_{k}\right) \leq \max _{x}\left\{-x \beta^{-1}(\alpha(x) / \varkappa)\right)+x \sigma_{k}\right\}=\max _{x}\left\{-x\left(\sigma_{k}-\beta^{-1}(\alpha(x) / \varkappa)\right)\right)\right\} .
$$


Hence, as in the proof of Proposition 5, it follows that

$$
\ln \mu\left(\sigma_{k}\right) \leq \sigma_{k} \alpha^{-1}\left(\varrho \beta\left(\sigma_{k}\right)\right)+O\left(\sigma_{k}\right), \quad k \rightarrow \infty,
$$

whence it follows that $\lambda_{\alpha \beta}^{M}(\ln \mu) \leq \varkappa$. In view of the arbitrariness of $\varkappa$ Proposition 7 is proved.

Proposition 8. Let $\alpha \in L^{0}, \beta \in L^{0}, \varrho_{\alpha \beta}^{M}(\ln \mu)<+\infty$, the function $F \in V$ satisfies condition (4) and $f$ has regular variation in regard to $F$. Then $\lambda_{\alpha \beta}^{M}(\ln \mu)=\lambda_{\alpha \beta}^{M}(I)$.

Indeed, since $f$ has regular variation in regard to $F$, from (5) we obtain $\lambda_{\alpha \beta}^{M}(\ln \mu) \leq$ $\lambda_{\alpha \beta}^{M}(I)$. On the other hand, since $\varrho_{\alpha \beta}^{M}(\ln \mu)<+\infty$, we have $(6)$ for every $\varepsilon \in(0,1)$ and all $\sigma \geq \sigma_{0}(\varepsilon)$. Since $\alpha \in L^{0}$ and $\beta \in L^{0}$, hence $\lambda_{\alpha \beta}^{M}(I) \leq \lambda_{\alpha \beta}^{M}(\ln \mu)$. Thus, $\lambda_{\alpha \beta}^{M}(\ln \mu)=$ $=\lambda_{\alpha \beta}^{M}(I)$.

From Propositions 7 and 8 we obtain the following theorem.

Theorem 4. Let either $\alpha \in L_{s i}$ and $\beta \in L^{0}$ or $\alpha \in L^{0}$ and $\beta \in L_{s i}$ and the function $F \in V$ satisfies condition (4). Suppose that $f$ has regular variation in regard to $F$ and $v(x)=-(\ln f(x))^{\prime} \uparrow+\infty$ as $x_{0} \leq x \rightarrow+\infty$. Then $\lambda_{\alpha \beta}^{M}(I)=\varkappa_{\alpha \beta}(f)$.

4. Analogues of Theorem A. Let $L S(F)$ be the class of Laplace-Stieltjes integrals for which $\sigma_{c}=\sigma_{\mu}=+\infty$. Suppose that $I_{j} \in L S(F), 1 \leq j \leq m$, and

$$
I_{j}(\sigma)=\int_{0}^{\infty} f_{j}(x) e^{x \sigma} d F(x), \quad \sigma \in \mathbb{R} .
$$

The following theorem is an analogue of Theorem A.

Theorem 5. Let functions $\alpha \in L_{s i}$ and $\beta \in L^{0}$ satisfy condition (3) and the function $F \in V$ satisfy condition (4). Suppose that all functions $f_{j}$ have regular variation in regard to $F$ and $v_{j}(x)=-\left(\ln f_{j}(x)\right)^{\prime}$ is continuous and increasing on $\left[x_{0},+\infty\right)$. Also suppose that $f$ have regular variation in regard to $F$ and

$$
\beta\left(\frac{1}{x} \ln \frac{1}{f(x)}\right)=(1+o(1)) \prod_{j=1}^{m} \beta\left(\frac{1}{x} \ln \frac{1}{f_{j}(x)}\right)^{\omega_{j}}, \quad x \rightarrow+\infty,
$$

where $\omega_{j}>0$ for $1 \leq j \leq m$ and $\sum_{1 \leq j \leq m} \omega_{j}=1$.

If all integrals (10) have regular $\alpha \beta$-growth (i.e. $\lambda_{\alpha \beta}\left(I_{j}\right)=\varrho_{\alpha \beta}\left(I_{j}\right)<+\infty$ ) then integral (1) has regular $\alpha \beta$-growth and $\varrho_{\alpha \beta}(I)=\prod_{j=1}^{m}\left(\varrho_{\alpha \beta}\left(I_{j}\right)\right)^{\omega_{j}}$.

Proof. By Theorem $1 \varrho_{\alpha \beta}\left(I_{j}\right)=k_{\alpha \beta}\left(f_{j}\right)$ and by Theorem $2 \lambda_{\alpha \beta}\left(I_{j}\right)=\varkappa_{\alpha \beta}\left(f_{j}\right)$. Since $\lambda_{\alpha \beta}\left(I_{j}\right)=\varrho_{\alpha \beta}\left(I_{j}\right)=\varrho_{j}<1$, we have $k_{\alpha \beta}\left(f_{j}\right)=\varkappa_{\alpha \beta}\left(f_{j}\right)=\varrho_{j}$, that is

$$
\lim _{x \rightarrow+\infty} \frac{\alpha(x)}{\beta\left(\frac{1}{x} \ln \frac{1}{f_{j}(x)}\right)}=\varrho_{j} .
$$

Therefore, from (11) we obtain

$$
\lim _{x \rightarrow+\infty} \frac{1}{\alpha(x)} \beta\left(\frac{1}{x} \ln \frac{1}{f(x)}\right)=\lim _{x \rightarrow+\infty} \frac{1}{\alpha(x)} \prod_{j=1}^{m} \beta\left(\frac{1}{x} \ln \frac{1}{f_{j}(x)}\right)^{\omega_{j}}=
$$




$$
=\lim _{x \rightarrow+\infty} \prod_{j=1}^{m}\left(\frac{1}{\alpha(x)} \beta\left(\frac{1}{x} \ln \frac{1}{f_{j}(x)}\right)\right)^{\omega_{j}}=\prod_{j=1}^{m} \lim _{x \rightarrow+\infty}\left(\frac{1}{\alpha(x)} \beta\left(\frac{1}{x} \ln \frac{1}{f_{j}(x)}\right)\right)^{\omega_{j}}=\prod_{j=1}^{m}\left(\frac{1}{\varrho_{j}}\right)^{\omega_{j}}
$$

i. e.

$$
\prod_{j=1}^{m} \varrho_{j}^{\omega_{j}}=\lim _{x \rightarrow+\infty} \frac{\alpha(x)}{\beta\left(\frac{1}{x} \ln \frac{1}{f(x)}\right)}=k_{\alpha \beta}(f)=\varkappa_{\alpha \beta}(f) .
$$

By Propositions 2 and $1 \lambda_{\alpha \beta}(\ln I) \geq \lambda_{\alpha \beta}(\ln \mu) \geq \varkappa_{\alpha \beta}(f)$ and by Theorem $1 \varrho_{\alpha \beta}(\ln I)=$ $k_{\alpha \beta}(f)$. Therefore, $\varrho_{\alpha \beta}(I)=\lambda_{\alpha \beta}(I)=\prod_{j=1}^{m}\left(r_{\alpha \beta}\left(I_{j}\right)\right)^{\omega_{j}}$.

If we choose $\alpha(x)=\ln x$ and $\beta(x)=x$ for $x \geq x_{0}$ then from the definitions of $\varrho_{\alpha \beta}(I)$ and $\lambda_{\alpha \beta}(I)$ we obtain the definitions of the $R$-order $\varrho_{R}$ and lower $R$-order $\lambda_{R}$, respectively. Choosing some more $m=2$ and $\omega_{1}=\omega_{2}=1 / 2$, we get the following statement.

Corollary 1. Let $F \in V$ and $\ln F(x)=o(x \ln x)$ as $x \rightarrow+\infty$. Suppose that the functions $f_{j}, j=1,2$, have regular variation in regard to $F$ and $v_{j}(x)=-\left(\ln f_{j}(x)\right)^{\prime}$ is continuous and increasing on $\left[x_{0},+\infty\right)$. Also suppose that $f$ have regular variation in regard to $F$ and

$$
\ln \frac{1}{f(x)}=(1+o(1)) \sqrt{\ln \frac{1}{f_{1}(x)} \ln \frac{1}{f_{2}(x)}}, \quad x \rightarrow+\infty
$$

If integrals $I_{1}$ and $I_{2}$ have regular $R$-growth (i.e. $\lambda_{R}\left(I_{j}\right)=\varrho_{R}\left(I_{j}\right)<+\infty$ for $j=1,2$ ) then integral (1) has regular $R$-growth and $\varrho_{R}(I)=\sqrt{\varrho_{R}\left(I_{1}\right) \varrho_{R}\left(I_{1}\right)}$.

Using modified generalized orders we get the following theorem.

Theorem 6. Let either $\alpha \in L_{s i}$ and $\beta \in L^{0}$ or $\alpha \in L^{0}$ and $\beta \in L_{\text {si }}$, and the function $F \in V$ satisfy condition (4). Suppose that $f$ has regular variation in regard to $F$ and $v(x)=-(\ln f(x))^{\prime}$ is continuous and increasing on $\left[x_{0},+\infty\right)$. Also suppose that $f$ have regular variation in regard to $F$ and (11) holds.

If all integrals (10) have regular modified $\alpha \beta$-growth (i.e. $\lambda_{\alpha \beta}^{M}\left(I_{j}\right)=\varrho_{\alpha \beta}^{M}\left(I_{j}\right)<+\infty$ ) then integral (1) has regular modified $\alpha \beta$-growth and $\varrho_{\alpha \beta}^{M}(I)=\prod_{j=1}^{m}\left(\varrho_{\alpha \beta}^{M}\left(I_{j}\right)\right)^{\omega_{j}}$.

Proof. By Theorem $3 \varrho_{\alpha \beta}^{M}\left(I_{j}\right)=k_{\alpha \beta}\left(f_{j}\right)$ and by Theorem $4 \lambda_{\alpha \beta}^{M}\left(I_{j}\right)=\varkappa_{\alpha \beta}\left(f_{j}\right)$. Since $\lambda_{\alpha \beta}^{M}\left(I_{j}\right)=\varrho_{\alpha \beta}^{M}\left(I_{j}\right)=\varrho_{j}<1$, we have $k_{\alpha \beta}\left(f_{j}\right)=\varkappa_{\alpha \beta}\left(f_{j}\right)=\varrho_{j}$, that is (12) holds. Therefore, as in the proof of Theorem 5 we obtain (13) from (11). By Propositions 7 and $6 \lambda_{\alpha \beta}^{M}(\ln I) \geq$ $\lambda_{\alpha \beta}^{M}(\ln \mu) \geq \varkappa_{\alpha \beta}(f)$ and by Theorem $3 \varrho_{\alpha \beta}^{M}(\ln I)=k_{\alpha \beta}(f)$. Therefore, $\varrho_{\alpha \beta}^{M}(I)=\lambda_{\alpha \beta}(I)=$ $\prod_{j=1}^{m}\left(r_{\alpha \beta}^{M}\left(I_{j}\right)\right)^{\omega_{j}}$.

If we choose $\alpha(x)=\ln x$ and $\beta(x)=\ln x$ for $x \geq x_{0}$ then from the definitions of $\varrho_{\alpha \beta}(I)$ and $\lambda_{\alpha \beta}(I)$ we obtain the definitions of the logarithmic order $\varrho_{l}$ and lower logarithmic order $\lambda_{l}$, respectively. Since

$$
\frac{1}{\ln \sigma} \ln \left(\frac{\ln I(\sigma))}{\sigma}\right)=\frac{\ln \ln I(\sigma)}{\ln \sigma}-1
$$

for such function we have $\varrho_{\alpha \beta}^{M}(I)=\varrho_{l}(I)$ and $\lambda_{\alpha \beta}^{M}(I)=\lambda_{l}(I)$. Therefore, choosing $m=2$ and $\omega_{1}=\omega_{2}=1 / 2$, we get the following statement. 
Corollary 2. Let $F \in V$ and $\ln F(x)=o(x)$ as $x \rightarrow+\infty$. Suppose that the functions $f_{j}$, $j=1,2$, have regular variation in regard to $F$ and $v_{j}(x)=-\left(\ln f_{j}(x)\right)^{\prime}$ is continuous and increasing on $\left[x_{0},+\infty\right)$. Also suppose that $f$ has regular variation in regard to $F$ and

$$
\ln \left(\frac{1}{x} \ln \frac{1}{f(x)}\right)=(1+o(1)) \sqrt{\ln \left(\frac{1}{x} \ln \frac{1}{f_{1}(x)}\right) \ln \left(\frac{1}{x} \ln \frac{1}{f_{2}(x)}\right)}, x \rightarrow+\infty .
$$

If integrals $I_{1}$ and $I_{2}$ have regular logarithmic growth (i.e. $\lambda_{l}\left(I_{j}\right)=\varrho_{l}\left(I_{j}\right) \in(1,+\infty)$ for $j=$ $1,2)$ then integral $(1)$ has regular logarithmic growth and $\varrho_{l}(I)=\sqrt{\left(\varrho_{l}\left(I_{1}\right)-1\right)\left(\varrho_{l}\left(I_{1}\right)-1\right.}$.

Finally, if we choose $\alpha(x)=x$ and $\beta(x)=\ln x$ for $x \geq x_{0}$ then $\varrho_{\alpha \beta}^{M}(I)=T(I):=$ $=\varlimsup_{\sigma \rightarrow+\infty} \frac{\ln I(\sigma)}{\sigma \ln \sigma}$ and $\lambda_{\alpha \beta}^{M}(I)=t(I):=\varliminf_{\sigma \rightarrow+\infty} \frac{\ln I(\sigma)}{\sigma \ln \sigma}$, and we obtain the next corollary.

Corollary 3. Let $F \in V$ and $\ln F(x)=o\left(x^{2}\right)$ as $x \rightarrow+\infty$. Suppose that the functions $f_{j}$, $j=1,2$, have regular variation in regard to $F$ and $v_{j}(x)=-\left(\ln f_{j}(x)\right)^{\prime}$ is continuous and increasing on $\left[x_{0},+\infty\right)$. Also suppose that $f$ has regular variation in regard to $F$ and $(14)$ holds. If $t\left(I_{j}\right)=T\left(I_{j}\right)<+\infty$ for $j=1,2$ then for integral $(1) t(I)=T(I)=\sqrt{T\left(I_{1}\right) T\left(I_{1}\right)}$.

5. Analogues of Theorem B. Since $\varrho_{\alpha \beta}(I)=\varlimsup_{\sigma \rightarrow+\infty} \frac{\ln \exp \{\alpha(\ln I(\sigma))\}}{\ln \exp \{\beta(\sigma)\}}$, we define the generalized type $T_{\alpha \beta}(I)$ of integral (1) by the formula

$$
T_{\alpha \beta}(I)=\varlimsup_{\sigma \rightarrow+\infty} \frac{\exp \{\alpha(\ln I(\sigma))\}}{\exp \{\varrho \beta(\sigma)\}}, \quad\left(\varrho=\varrho_{\alpha \beta}(I)\right) .
$$

Theorem 1 implies the following lemma.

Lemma 2. Suppose that the functions $\alpha \in L$ and $\beta \in L$ are continuously differentiable, $x \alpha^{\prime}(x)=o(1), x \beta^{\prime}(x)=O(1)$ as $x \rightarrow+\infty$, and for every $c \in(-\infty,+\infty)$

$$
\frac{d \beta^{-1}(\alpha(x)+c)}{d \ln x}=O(1), \quad x \rightarrow+\infty
$$

If $F \in V, f$ has regular variation in regard to $F$ and for every $c \in(-\infty,+\infty)$

$$
\ln F(x)=o\left(x \beta^{-1}(\alpha(x)+c)\right), \quad x \rightarrow+\infty,
$$

then

$$
T_{\alpha \beta}(I)=\varlimsup_{x \rightarrow+\infty} \frac{\exp \{\alpha(x)\}}{\exp \left\{\beta\left(\frac{1}{x} \ln \frac{1}{f(x)}\right)\right\}} .
$$

Indeed, if $\alpha_{1} \in L$ and $\frac{x \alpha_{1}^{\prime}(x)}{\alpha_{1}(x)}=o(1)$ as $x \rightarrow+\infty$ then $\alpha_{1} \in L_{s i}$, and if $\beta_{1} \in L$ and $\frac{x \beta_{1}^{\prime}(x)}{\beta_{1}(x)}=O(1)$ as $x \rightarrow+\infty$ then $\beta_{1} \in L^{0}$. Hence it follows that if $\alpha_{1}(x)=e^{\alpha(x)}, \beta_{1}(x)=e^{\beta(x)}$ and $x \alpha^{\prime}(x)=o(1), x \beta^{\prime}(x)=O(1)$ as $x \rightarrow+\infty$ then $\alpha_{1} \in L_{s i}$ and $\beta_{1} \in L^{0}$. From (15) condition (3) follows with $\alpha_{1}$ and $\beta_{1}$ instead of $\alpha$ and $\beta$. Condition (18) implies (4) with $\alpha_{1}$ and $\beta_{1}$ instead of $\alpha$ and $\beta$. Therefore, Theorem 1 implies Lemma 2. 
Theorem 7. Let functions $\alpha \in L$ and $\beta \in L$ be continuously differentiable, $x \alpha^{\prime}(x)=$ $o(1), x \beta^{\prime}(x)=O(1)$ as $x \rightarrow+\infty$ and (3) and (15) hold. Let $F \in V, f$ and $f_{j}$ have a regular variation in regard to $F$ and (18) holds. Suppose that all integrals (10) have the same generalized order $\varrho_{\alpha \beta}\left(I_{j}\right)=\varrho \in(0,+\infty)$ and the generalized types $T_{\alpha \beta}\left(I_{j}\right) \in(0,+\infty)$. Suppose also that $f_{1}(x)>0$ for all $x \geq x_{0}$ and for all $2 \leq j \leq m$

$$
\beta\left(\frac{1}{x} \ln \frac{1}{f_{j}(x)}\right) \leq(1+o(1)) \beta\left(\frac{1}{x} \ln \frac{1}{f_{1}(x)}\right), \quad x \rightarrow+\infty .
$$

If $\omega_{j}>0$ for $1 \leq j \leq m, \sum_{1 \leq j \leq m} \omega_{j}=1$ and

$$
\exp \left\{\beta\left(\frac{1}{x} \ln \frac{1}{f(x)}\right)\right\}=(1+o(1)) \prod_{j=1}^{m} \exp \left\{\omega_{j} \beta\left(\frac{1}{x} \ln \frac{1}{f_{j}(x)}\right)\right\}, \quad x \rightarrow+\infty
$$

then integral (1) has the generalized order $\varrho_{\alpha \beta}(I)=\varrho$ and the generalized type $T_{\alpha \beta}(I) \leq$ $\prod_{j=1}^{m} T_{\alpha \beta}\left(I_{j}\right)^{\omega_{j}}$.

Proof. At first, we remark that from the conditions $x \alpha^{\prime}(x)=o(1), x \beta^{\prime}(x)=O(1)$ as $x \rightarrow+\infty$ it follows that $\alpha \in L_{s i}$ and $\beta \in L^{0}$, and (18) implies (4). Thus, the functions $\alpha, \beta$, and $F$ satisfy the assumptions of Theorem 1 .

From (18) we have

$$
\beta\left(\frac{1}{x} \ln \frac{1}{f(x)}\right)=\sum_{j=1}^{m} \omega_{j} \beta\left(\frac{1}{x} \ln \frac{1}{f_{j}(x)}\right)+o(1), \quad x \rightarrow+\infty .
$$

Therefore, by Theorem 1

$$
\frac{1}{\varrho_{\alpha \beta}(I)}=\varliminf_{x \rightarrow+\infty} \frac{1}{\alpha(x)} \beta\left(\frac{1}{x} \ln \frac{1}{f(x)}\right) \geq \sum_{j=1}^{m} \underset{x \rightarrow+\infty}{\lim } \frac{\omega_{j}}{\alpha(x)} \beta\left(\frac{1}{x} \ln \frac{1}{f_{j}(x)}\right)=\frac{1}{\varrho} .
$$

On the other hand, in view of (17) we obtain from (19)

$$
\frac{1}{\varrho_{\alpha \beta}(I)} \leq \sum_{j=1}^{m} \underline{\lim _{x \rightarrow+\infty}} \frac{\omega_{j}}{\alpha(x)} \beta\left(\frac{1}{x} \ln \frac{1}{f_{1}(x)}\right)=\frac{1}{\varrho} .
$$

Thus, $\varrho_{\alpha \beta}(I)=\varrho$.

From (18) and Lemma 2 it follows that

$$
\begin{aligned}
& \frac{1}{T_{\alpha \beta}(I)}=\varliminf_{x \rightarrow+\infty} \frac{1}{\exp \alpha(x)} \prod_{j=1}^{m} \exp \left\{\varrho \omega_{j} \beta\left(\frac{1}{x} \ln \frac{1}{f_{j}(x)}\right)\right\} \geq \\
\geq & \prod_{j=1}^{m} \underline{\lim _{x \rightarrow+\infty}}\left(\frac{\exp \left\{\varrho \omega_{j} \beta\left(\frac{1}{x} \ln \frac{1}{f_{j}(x)}\right)\right\}}{\exp \alpha(x)}\right)^{\omega_{j}}=\prod_{j=1}^{m}\left(\frac{1}{T_{\alpha \beta}(I)}\right)^{\omega_{j}} .
\end{aligned}
$$

If we choose $\alpha(x)=\ln \ln \ln x, \beta(x)=\ln x$ for $x \geq x_{0}, m=2$ and $\omega_{j}=1 / 2$ then from Theorem 7 we obtain the following statement. 
Corollary 4. Let $F \in V, \ln F(x)=o(x \ln \ln x)$ as $x \rightarrow+\infty$ and the functions $f$ and $f_{j}$ $(j=1,2)$ have regular variation in regard to $F$. Suppose that $f_{1}(x)>0$ for all $x \geq x_{0}$ and $\ln \left(\frac{1}{x} \ln \frac{1}{f_{2}(x)}\right) \leq(1+o(1)) \ln \left(\frac{1}{x} \ln \frac{1}{f_{1}(x)}\right)$ as $x \rightarrow+\infty$.

If $\varlimsup_{\sigma \rightarrow+\infty} \frac{\ln \ln \ln \ln I_{j}(\sigma)}{\ln \sigma}=\varrho \in(0,+\infty)$ for $j=1,2$ and

$$
\ln \frac{1}{f(x)}=(1+o(1)) \sqrt{\ln \frac{1}{f_{1}(x)} \ln \frac{1}{f_{2}(x)}}, \quad x \rightarrow+\infty,
$$

then $\varlimsup_{\sigma \rightarrow+\infty} \frac{\ln \ln \ln \ln I(\sigma)}{\ln \sigma}=\varrho$ and

$$
\varlimsup_{x \rightarrow+\infty} \frac{x}{\exp _{3}\left\{\left(\frac{1}{x} \ln \frac{1}{f(x)}\right)^{\varrho}\right\}} \leq \sqrt{\varlimsup_{x \rightarrow+\infty} \frac{x}{\exp _{3}\left\{\left(\frac{1}{x} \ln \frac{1}{f(x)}\right)^{\varrho}\right\}} \varlimsup_{x \rightarrow+\infty} \frac{x}{\exp _{3}\left\{\left(\frac{1}{x} \ln \frac{1}{f(x)}\right)^{\varrho}\right\}}}
$$

where $\left.\exp _{3} x=\exp \left\{\exp \left\{e^{x}\right\}\right\}\right\}$.

For integral (1) of finite modified generalized orders we define the generalized type $T_{\alpha \beta}^{M}(I)$ by the formula

$$
T_{\alpha \beta}^{M}(I)=\varlimsup_{\sigma \rightarrow+\infty} \frac{\ln I(\sigma))}{\sigma \alpha^{-1}(\varrho \beta(\sigma))}, \quad\left(\varrho=\varrho_{\alpha \beta}^{M}(I)\right) .
$$

Then the following lemma is true.

Lemma 3. Let $\beta \in L, \beta_{1}(x)=\alpha^{-1}(\varrho \beta(x)) \in L_{\text {si }}$, the function $F \in V$ satisfy condition $\ln F(x)=o\left(x \beta_{1}^{-1}(c x)\right)$ as $x \rightarrow+\infty$ for every $c \in(0,+\infty)$, and $f$ have regular variation in regard to $F$. Then

$$
T_{\alpha \beta}^{M}(I)=\varlimsup_{x \rightarrow+\infty} \frac{x}{\alpha^{-1}\left(\varrho \beta\left(\frac{1}{x} \ln \frac{1}{f(x)}\right)\right)} .
$$

Indeed, $\left.\beta_{1}^{-1}(c x)\right)=\beta^{-1}(c \alpha(x / \varrho)) \leq \beta^{-1}\left(\alpha(x) / \varrho_{1}\right)$, because from the condition $\alpha \in L^{0}$ it follows that $\alpha(c x) \leq K(c) \alpha(c x)$ for every $c \in(0,+\infty)$. Therefore, if we choose $\alpha_{1}(x) \equiv x$ for $x \geq x_{0}$, then from Theorem 3 with $\alpha_{1}$ and $\beta_{1}$ instead of $\alpha$ and $\beta$ we deduce Lemma 3 .

Theorem 8. Let $\beta \in L_{s i}, \alpha(x)=(1+o(1)) \ln x$ as $x \rightarrow+\infty$ and $\beta_{1}(x)=\alpha^{-1}(\varrho \beta(x)) \in L_{s i}$. Let $F \in V, \ln F(x)=o\left(x \beta_{1}^{-1}(x)\right)$ as $x \rightarrow+\infty$ and $f$ and $f_{j}$ have regular variation in regard to F. Suppose that all integrals (10) have the same modified generalized order $\varrho_{\alpha \beta}^{M}\left(I_{j}\right)=\varrho \in$ $(0,+\infty)$ and the modified generalized types $T_{\alpha \beta}^{M}\left(I_{j}\right) \in(0,+\infty)$. Suppose also that $f_{1}(x)>0$ for all $x \geq x_{0}$ and (17) holds.

$$
\begin{aligned}
& \text { If } \omega_{j}>0 \text { for } 1 \leq j \leq m, \sum_{1 \leq j \leq m} \omega_{j}=1 \text {, and } \\
& \alpha^{-1}\left(\varrho \beta\left(\frac{1}{x} \ln \frac{1}{f(x)}\right)\right)=(1+o(1)) \prod_{j=1}^{m}\left(\alpha^{-1}\left(\varrho \beta\left(\frac{1}{x} \ln \frac{1}{f_{j}(x)}\right)\right)\right)^{\omega_{j}}, \quad x \rightarrow+\infty,
\end{aligned}
$$

then integral (1) has the modified generalized order $\varrho_{\alpha \beta}^{M}(I)=\varrho$ and the modified generalized type $T_{\alpha \beta}^{M}(I) \leq \prod_{j=1}^{m} T_{\alpha \beta}^{M}\left(I_{j}\right)^{\omega_{j}}$. 
Proof. At first, we remark that the condition $\ln F(x)=o\left(x \beta_{1}^{-1}(x)\right)$ as $x \rightarrow+\infty$ implies (4), because $\left.\left.\beta_{1}^{-1}(x)\right)=\beta^{-1}(\alpha(t) / \varrho)\right)$. Thus, the functions $\alpha, \beta$, and $F$ satisfy the conditions of Theorem 3 .

Since $\alpha(x)=(1+o(1)) \ln x$ as $x \rightarrow+\infty$, from (20) we obtain (19). As above from (19) by Theorem $31 / \varrho_{\alpha \beta}^{M}(I) \geq 1 / \varrho$. On the other hand, from (17) and (19) as above we get $1 / \varrho_{\alpha \beta}^{M}(I) \leq 1 / \varrho$. Thus, $\varrho_{\alpha \beta}^{M}(I)=\varrho$.

From (20) and Lemma 3 it follows that

$$
\begin{gathered}
\frac{1}{T_{\alpha \beta}^{M}(I)}=\varliminf_{x \rightarrow+\infty} \frac{1}{x} \alpha^{-1}\left(\varrho \beta\left(\frac{1}{x} \ln \frac{1}{f(x)}\right)\right)=\varliminf_{x \rightarrow+\infty} \frac{1}{x} \prod_{j=1}^{m}\left(\alpha^{-1}\left(\varrho \beta\left(\frac{1}{x} \ln \frac{1}{f_{j}(x)}\right)\right)\right)^{\omega_{j}} \geq \\
=\prod_{j=1}^{m} \underline{\lim _{x \rightarrow+\infty}}\left(\frac{1}{x} \alpha^{-1}\left(\varrho \beta\left(\frac{1}{x} \ln \frac{1}{f_{j}(x)}\right)\right)\right)^{\omega_{j}}=\prod_{j=1}^{m}\left(\frac{1}{T_{\alpha \beta}^{M}(I)}\right)^{\omega_{j}} .
\end{gathered}
$$

If we choose $\alpha(x)=\ln x, \beta(x)=\ln \ln x$ for $x \geq x_{0}, \mathrm{~m}=2$ and $\omega_{j}=1 / 2$ then from Theorem 8 we obtain the following statement.

Corollary 5. Let $F \in V, \ln F(x)=o\left(x \exp \left\{e^{x}\right\}\right)$ as $x \rightarrow+\infty$ and the functions $f$ and $f_{j}$, $j=1,2$, have regular variation in regard to $F$. Suppose that that $f_{1}(x)>0$ for all $x \geq x_{0}$ and $\ln \ln \left(\frac{1}{x} \ln \frac{1}{f_{2}(x)}\right) \leq(1+o(1)) \ln \ln \left(\frac{1}{x} \ln \frac{1}{f_{1}(x)}\right)$ as $x \rightarrow+\infty$.

If $\varlimsup_{\sigma \rightarrow+\infty} \frac{\ln \ln I_{j}(\sigma)-\ln \sigma}{\ln \ln \sigma}=\varrho \in(0,+\infty)$ for $j=1,2$ and

$$
\ln \left(\frac{1}{x} \ln \frac{1}{f(x)}\right)=(1+o(1)) \sqrt{\ln \left(\frac{1}{x} \ln \frac{1}{f_{1}(x)}\right) \ln \left(\frac{1}{x} \ln \frac{1}{f_{2}(x)}\right)}, \quad x \rightarrow+\infty,
$$

then $\varlimsup_{\sigma \rightarrow+\infty} \frac{\ln \ln I(\sigma)-\ln \sigma}{\ln \ln \sigma}=\varrho$ and $\varlimsup_{\sigma \rightarrow+\infty} \frac{\ln I(\sigma)}{\sigma \ln ^{\varrho} \sigma} \leq \sqrt{\varlimsup_{\sigma \rightarrow+\infty} \frac{\ln I_{1}(\sigma)}{\sigma \ln ^{\varrho} \sigma} \varlimsup_{\sigma \rightarrow+\infty} \frac{\ln I_{2}(\sigma)}{\sigma \ln ^{\varrho} \sigma}}$.

Finally, we prove a theorem, which supplements Theorems 6 and 8 .

Theorem 9. Let either $\alpha \in L_{s i}$ and $\beta \in L^{0}$ or $\alpha \in L^{0}$ and $\beta \in L_{s i}, F \in V, \ln F(x)=$ $=o\left(x \beta^{-1}\left(\alpha^{c}(x)\right)\right)$ as $x \rightarrow+\infty$ for every $c \in(0,+\infty)$ and integrals (10) have modified general orders $\varrho_{\alpha \beta}^{M}\left(I_{j}\right) \in(0,+\infty)$. Suppose that $f$ has regular variation in regard to $F$ and (11) holds. Then:

1) if $f_{1}(x)>0$ for all $x \geq x_{0}$ and for all $2 \leq j \leq m_{0}$

$$
\ln \beta\left(\frac{1}{x} \ln \frac{1}{f_{j}(x)}\right) \leq(1+o(1)) \ln \beta\left(\frac{1}{x} \ln \frac{1}{f_{1}(x)}\right), \quad x \rightarrow+\infty .
$$

then

$$
\varlimsup_{\sigma \rightarrow+\infty} \frac{1}{\ln \beta(\sigma)} \ln \alpha\left(\frac{\ln I(\sigma)}{\sigma}\right)=1
$$

and $\varrho_{\alpha \beta}^{M}(I) \leq \prod_{j=1}^{m}\left(\varrho_{\alpha \beta}^{M}\left(I_{j}\right)\right)^{\omega_{j}}$

2) if $v(x)=-(\ln f(x))^{\prime}$ is continuous and increasing on $\left[x_{0},+\infty\right)$ and all integrals $(10)$ have regular modified $\alpha \beta$-growth then integral (1) has regular modified $\alpha \beta$-growth and

$$
\varrho_{\alpha \beta}^{M}(I)=\prod_{j=1}^{m}\left(\varrho_{\alpha \beta}^{M}\left(I_{j}\right)\right)^{\omega_{j}}
$$


Proof. Since $\varrho_{\alpha \beta}^{M}\left(I_{j}\right) \in(0,+\infty)$, we have

$$
\varlimsup_{\sigma \rightarrow+\infty} \frac{1}{\ln \beta(\sigma)} \ln \alpha\left(\frac{\ln I_{j}(\sigma)}{\sigma}\right)=1
$$

It is known ([9]) that if $h \in L^{0}$ then $h$ is a $R O$-increase function ([10, p.86]), that is for every $\lambda \in[1,+\infty)$ and all $x \geq x_{0}$ the inequalities $1 \leq h(\lambda x) / h(x) \leq M(\lambda)<+\infty$, whence it follows that $\ln h \in L_{s i}$. Therefore, using Theorem 3 with $\ln \alpha$ and $\ln \beta$ instead of $\alpha$ and $\beta$ (the condition $\ln F(x)=o\left(x \beta^{-1}\left(\alpha^{c}(x)\right)\right)$ as $x \rightarrow+\infty$ for every $c \in(0,+\infty)$ implies condition (4)), we obtain

$$
\varliminf_{x \rightarrow+\infty} \frac{1}{\ln \alpha(x)} \ln \beta\left(\frac{1}{x} \ln \frac{1}{f_{j}(x)}\right)=1
$$

for each $j=1,2, \ldots, m$, and in view of (11)

$$
\begin{gathered}
\varliminf_{x \rightarrow+\infty} \frac{1}{\ln \alpha(x)} \ln \beta\left(\frac{1}{x} \ln \frac{1}{f(x)}\right)=\underline{\lim }_{x \rightarrow+\infty} \frac{1}{\ln \alpha(x)} \sum_{j=1}^{m} \omega_{j} \ln \beta\left(\frac{1}{x} \ln \frac{1}{f_{j}(x)}\right) \geq \\
\geq \sum_{j=1}^{m} \omega_{j} \underset{x \rightarrow+\infty}{\lim \alpha(x)}\left(\frac{1}{x} \ln \frac{1}{f_{1}(x)}\right)=1 .
\end{gathered}
$$

On the other hand by virtue of $(21)$

$$
\begin{gathered}
\varliminf_{x \rightarrow+\infty} \frac{1}{\ln \alpha(x)} \ln \beta\left(\frac{1}{x} \ln \frac{1}{f(x)}\right)= \\
=\varliminf_{x \rightarrow+\infty} \frac{1}{\ln \alpha(x)}\left(\omega_{1} \ln \beta\left(\frac{1}{x} \ln \frac{1}{f_{1}(x)}\right)+\sum_{j=2}^{m} \omega_{j} \ln \beta\left(\frac{1}{x} \ln \frac{1}{f_{j}(x)}\right)\right) \leq \\
\leq \varliminf_{x \rightarrow+\infty} \frac{1}{\ln \alpha(x)} \sum_{j=1}^{m} \omega_{j} \ln \beta\left(\frac{1}{x} \ln \frac{1}{f_{1}(x)}\right)=1
\end{gathered}
$$

i. e.

$$
\varlimsup_{x \rightarrow+\infty} \frac{\ln \alpha(x)}{\ln \beta\left(\frac{1}{x} \ln \frac{1}{f(x)}\right)}=1
$$

and by Theorem 3 equality (22) is true.

The condition $\ln F(x)=o\left(x \beta^{-1}\left(\alpha^{c}(x)\right)\right)$ as $x \rightarrow+\infty$ for every $c \in(0,+\infty)$ implies the condition ln $F(x)=o\left(x \beta^{-1}(\alpha(c x))\right)$ as $x \rightarrow+\infty$ for every $c \in(0,+\infty)$. Therefore, by Theorem 3 in view of $(11)$

$$
\begin{aligned}
\frac{1}{\varrho_{\alpha \beta}^{M}(I)}= & \varliminf_{x \rightarrow+\infty} \frac{1}{\alpha(x)} \beta\left(\frac{1}{x} \ln \frac{1}{f(x)}\right)=\varliminf_{x \rightarrow+\infty} \prod_{j=1}^{m}\left(\frac{1}{\alpha(x)} \beta\left(\frac{1}{x} \ln \frac{1}{f_{j}(x)}\right)\right)^{\omega_{j}} \geq \\
& \geq \prod_{j=1}^{m} \underline{\lim _{x \rightarrow+\infty}}\left(\frac{1}{\alpha(x)} \beta\left(\frac{1}{x} \ln \frac{1}{f_{j}(x)}\right)\right)^{\omega_{j}}=\prod_{j=1}^{m}\left(\varrho_{\alpha \beta}^{M}\left(I_{j}\right)\right)^{\omega_{j}},
\end{aligned}
$$

i. e. the statement 1) is proved. 
Now, if $\varrho_{\alpha \beta}^{M}\left(I_{j}\right)=\varrho_{\alpha \beta}^{M}\left(I_{j}\right)$ then by Theorem 4 for each $j=1,2, \ldots m$

$$
\frac{1}{\alpha(x)} \beta\left(\frac{1}{x} \ln \frac{1}{f_{j}(x)}\right) \rightarrow \frac{1}{\varrho_{\alpha \beta}^{M}\left(I_{j}\right)}, \quad x \rightarrow+\infty,
$$

and from (11) we get

$$
\begin{gathered}
\frac{1}{\alpha(x)} \beta\left(\frac{1}{x} \ln \frac{1}{f(x)}\right)=(1+o(1)) \prod_{j=1}^{m} \frac{1}{\alpha(x)} \beta\left(\frac{1}{x} \ln \frac{1}{f_{j}(x)}\right)= \\
=(1+o(1)) \prod_{j=1}^{m}\left(\frac{1}{\varrho_{\alpha \beta}^{M}\left(I_{j}\right)}\right)^{\omega_{j}}, \quad x \rightarrow+\infty .
\end{gathered}
$$

Hence by Theorems 3 and 4 it follows that integral (1) has regular modified $\alpha \beta$-growth and $\varrho_{\alpha \beta}^{M}(I)=\prod_{j=1}^{m}\left(\varrho_{\alpha \beta}^{M}\left(I_{j}\right)\right)^{\omega_{j}}$.

\section{REFERENCES}

1. E.G. Calys, A note on the order and type of integral functions, Riv. Mat. Univer Parma, 5 (1964), $133-137$.

2. R.S.L. Srivastava, On the order and type of integral functions, Riv. Mat. Univer Parma, 1 (1959), 249255.

3. R.S.L. Srivastava, On the order and type of integral functions, Riv. Mat. Univer Parma, 2 (1961), 265270.

4. L.V. Kulavec', O.M. Mulyava, On the growth of a class of entire Dirichlet series, Carpatian Math. Publ., 5 (2014) (2), 300-309. doi: 10.15330/cmp.6.2.300-309 (in Ukrainian).

5. O.B. Skaskiv, On certain relations between the maximum modulus and the maximal term of an entire Dirichlet series, Math. Notes 66 (1999), 223-232. doi.org/10.1007/BF02674881

6. O.B. Skaskiv, O.M. Trakalo, Asymptotic estimates for Laplace integrals, Mat. Stud., 18 (2002) (2), 125-146. (in Ukrainian)

7. M.M. Sheremeta, Asymptotical behaviour of Laplace-Stieltjes integrals, VNTL Publishers, Lviv, 2010.

8. A.J. Macintyre, Laplace's transformation and integral functions, Proc. London Math. Soc., 45 (1939), 1-20. doi.org/10.1112/plms/s2-45.1.1

9. M.M. Sheremeta, On two classes of positive functions and belonging to them of main characteristics of entire functions, Mat. Stud., 19 (2003) (1), 73-82.

10. E. Seneta, Regularly varying functions, Lect. Notes Math., 508, Springer-Verlag, Berlin, 1976.

Ivan Franko National University of Lviv, Ukraine m_m_sheremeta@gmail.com

andriykuryliak@gmail.com 